\title{
Basic Principles of Intraoperative Ultrasound Applied to Brain Tumor Surgery
}

\author{
Javier A. Jacobo ${ }^{1, \odot}$ Javier Avendaño ${ }^{1}$ Sergio Moreno-Jimenez ${ }^{1} \quad$ Santiago Nuñez ${ }^{1}$ Rocio Mamani ${ }^{1}$ \\ 1Department of Surgical Neuro-Oncology, National Institute of \\ Neurology and Neurosurgery, Mexico City, Mexico

\begin{abstract}
Address for correspondence Javier A. Jacobo, Department of Surgical Neuro-Oncology, National Institute of Neurology and Neurosurgery, Insurgentes Sur 3877, La Fama, Mexico City 14269, Mexico (e-mail: jacoboncx@gmail.com).
\end{abstract}

Indian J Neurosurg:2020;9:135-140

\begin{abstract}
Keywords

- basic principles

- ultrasound

- neurosurgery

- neuro-oncology

Intraoperative ultrasound (US) has been shown to possess great value in assessing tumor volume and localization, especially for primary resection of gliomas and metastatic lesions. Given that US is a technology that is highly user dependent, many surgeons have encountered problems with the usage of this technology, as well as interpretation of intraoperative US images, limiting its full potential. This article focuses on the basic knowledge a neurosurgeon must acquire to properly use and interpret intraoperative US to improve tumor localization and extent of resection during brain tumor surgery.
\end{abstract}

\section{Introduction}

Ultrasound (US) was first introduced in the 1920s by Loomis and Wood, who described its biological effects on living tissue. ${ }^{1}$ Since then, this technology has been applied to multiple fields of medicine including neurosurgery.

The first US usage in neurosurgery was reported by the Dussik brothers, who attempted to use it to identify brain tumors. ${ }^{2}$ It was not until 1978 that ultrasonography was first used to aid in the surgical resection of a central nervous system (CNS) tumor. ${ }^{3}$ Advances in ultrasound imaging have made it popular in the common practice in recent years, making it a very valuable tool for identifying the tumor and aiding in its total resection. ${ }^{4,5}$

This article will focus on the basic principles and techniques a neurosurgeon must know to perform an adequate US-guided surgery for the resection of a brain tumor, based on the experience acquired by the group of Surgical Neuro-oncology in the National Institute of Neurology and Neurosurgery.

\section{Basic Principles of Ultrasound}

Sound is created when a vibrating source comes in contact with a medium, causing it to vibrate. The mechanical energy, which is generated from the vibrations, travels in a longitudinal wave through the medium, generating cyclical areas of high and low pressure, known as compressions and rarefactions. US is considered as the frequencies that exceed the limits of human hearing, or $20 \mathrm{kHz}$. Modern US systems typically use frequencies between 2 and $10 \mathrm{MHz} .{ }^{6}$ Frequencies differ when different tissues are targeted; greater frequencies offer better imaging at the surface, while lower frequencies are used when a target lies deeper within the tissue.

Sound travels at different speeds through different media. In diagnostic US, human tissue is the medium in which sound travels. By comparison, the propagation velocity of air is quite slow, $440 \mathrm{~m} / \mathrm{s}$, and in bone, it can be as high as $5,000 \mathrm{~m} / \mathrm{s}$. The basis of diagnostic US is the pulse-echo technique in which small bursts of sound are transmitted into the tissue, and the reflected echoes are then measured. ${ }^{7,8}$

As previously mentioned, US relies on echoes emitted by a transducer, which are then reflected by various tissue interfaces of different density. The intensity of the echo received by the same transducer is used to calculate the brightness of the specific reflector, whereas the position and space is defined by the time sound needs to travel from emission to reception. This means that the image recreated on the screen is the result of calculations done by the software of the machine, rather than a photograph being projected. ${ }^{79}$
DOI https://doi.org/

10.1055/s-0040-1705289

ISSN 2277-954X.
(C2020 Neurological Surgeons'

Society of India
License terms

()(1) $\Theta \circledast$ 
The received echoes can be displayed in the following two modes: A-Mode which is projected in a linear graph, or the so-called brightness mode or B-Mode, which displays the brightness of the echoes throughout an entire section; fast image update in a real-time fashion provides a film-like presentation of a cross-sectional view of the imaged region. ${ }^{9}$

\section{Indications for Intraoperative Ultrasound}

Just like many other technologies, intraoperative US should be used with rationale and applied to specific cases.

In neuro-oncology, there are three main applications: tumor localization, evaluation of the extent of resection, and assessment of vascular supply or patency of venous sinuses.

All of these indications will be discussed in detail as the article goes on.

\section{Imaging Interpretation and Technique for Adequate Image Acquisition}

The echogenicity of the tissue refers to the ability to reflect or transmit US waves in the context of surrounding tissues. ${ }^{10}$ Whenever there is an interface of structures with different echogenicities, a visible difference in contrast will be apparent on the screen. Based on echogenicity, a structure can be characterized as hyperechoic (white on the screen), hypoechoic (gray on the screen), and anechoic (black on the screen). ${ }^{11}$ In the gray spectrum that appears on the screen on B-Mode, the softer the medium is, the darker the image will appear (-Fig. 1). This way, it is possible to differ from cystic lesions all the way to calcified lesions within the brain parenchyma.

Some structures are easily recognizable because of their distinct echogenicity; naturally hyperechoic structures are the dural folds, choroid plexus, and pineal gland (depending on degree of calcification). Normal brain tissue will be isoechogenic. Hypoechogenic and anechogenic structures include the brainstem and cerebrospinal fluid (CSF)-filled cavities.

One must keep in mind that during surgery, abnormal tissue will be encountered. Lesions like solid tumors,

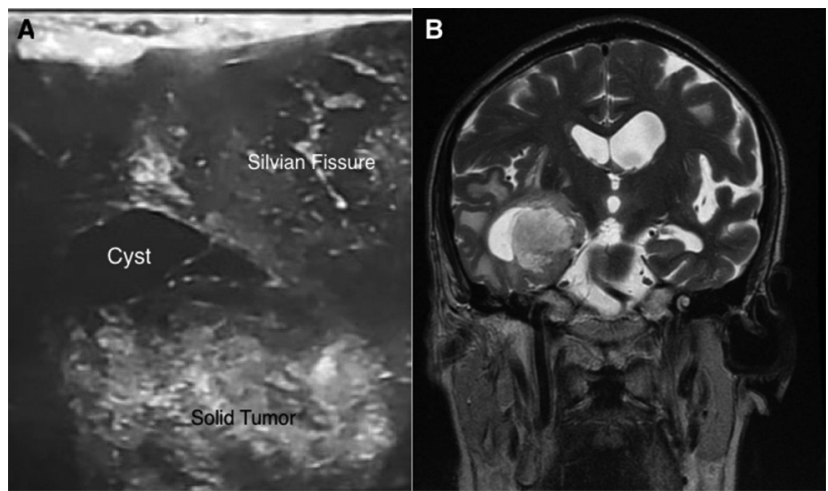

Fig. 1 Intraoperative US image (A) and preoperative MRI (B). The lesion had a cystic portion that in US is viewed as hypoechogenic; the solid part of the tumor is hyperechoic with some calcified portions that can be identified because of its brighter color on the screen. MRI, magnetic resonance imaging; US, ultrasound. calcifications, and some gliomas will appear hyperechogenic in relation to healthy tissue on the screen (-Fig. 2). Cystic lesions or the necrotic part of a glioblastoma will appear hypoechogenic in relation to healthy tissue ( - Fig. 3)..$^{12-14}$

Adequate technique and placement of the transducer is of crucial importance to reliably discriminate the images obtained through the procedure.

Choosing of the probe for the desired space and lesion is important; the most commonly used probe in cranial surgery is a $30 \times 12 \mathrm{~mm}$ probe with frequency of $7 \mathrm{MHz}$, as this provides a good balance between resolution and penetration. ${ }^{12}$ It is important to state that the craniotomy should be of a large enough size to accommodate the probe and facilitate the technique.

Before starting, some default settings are helpful in obtaining a better image during the procedure. Initiate by activating the "tissue harmonic intensity" option to improve image quality, then adjust image gain until the region of interest shows optimal brightness; finally, adjust the zoom on the device, start with zoom out, and then gradually zoom in until the image on the screen is large enough. ${ }^{12}$

After choosing the adequate probe and the US setting is in place, the next step is to adequately place the transducer.

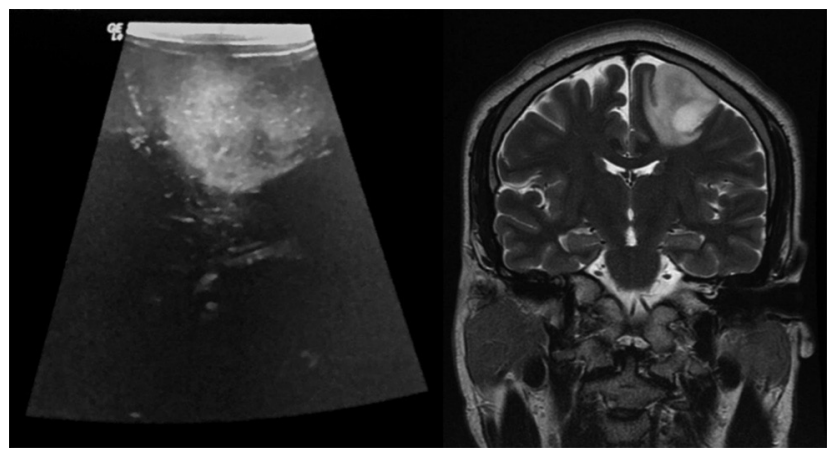

Fig. 2 Intraoperative US image of a frontal low-grade glioma; note its hyperchogenicity compared with the healthy surrounding tissue. When compared with the preoperative MRI, it shows adequate anatomical similarity. To obtain this image, the transducer is placed perpendicular to the midline giving a coronal view. MRI, magnetic resonance imaging; US, ultrasound. 
It should be held so that the external notch is facing the patient's anatomic right in a cross-sectional view or toward the head in a longitudinal view. ${ }^{7}$ In this way, the surgeon can reliably assess where the lesion is displayed in the B-Mode and also make adjustments during the procedure.

An important concept to keep in mind is the angle of incidence; this is the angle at which the waves encounter the surface of the examined tissue. ${ }^{11}$ The more perpendicular the transducer is placed to the surface of the brain, the less waves will be scattered and the better the image will be seen on the screen. Image resolution can be improved by tilting the transducer, thus adjusting the angle of incidence.

The morphology of the image will change depending on the position of the transducer in relation to the surface of the brain. The scanning planes are similar to the familiar anatomical planes; if the transducer in placed in a horizontal position in the lateral convexity of the brain, the obtained image will resemble of that of an axial view in the magnetic resonance imaging (MRI) ( $\boldsymbol{\sim}$ Fig. 4 ). If the transducer is positioned vertically in the lateral convexity, a somewhat coronal

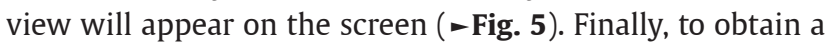
sagittal view, the transducer would have to be located parallel to the sagittal sinus in the superior convexity of the surface of the brain (-Fig. 6).

The depth of the target lesion within the brain is a variable that must be considered in choosing the right probe for the study. High-frequency probes will give better resolution for
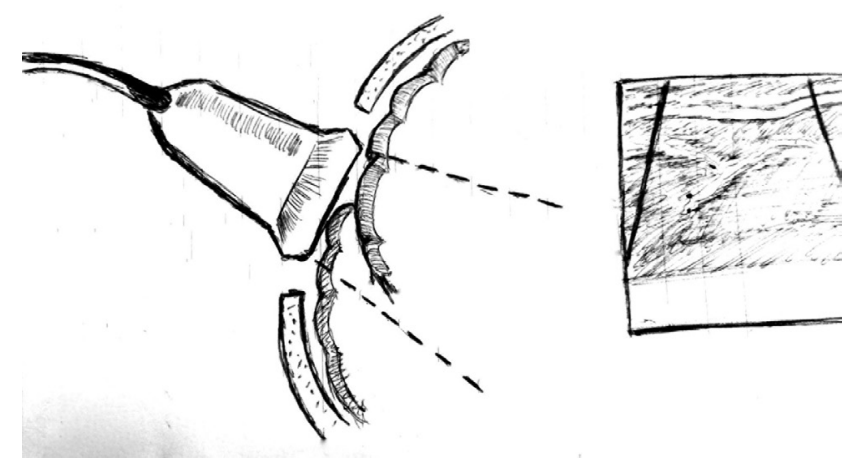

Fig. 4 Schematic representation to obtain an axial view with intraoperative US. The probe is positioned horizontally parallel to the skull base on the lateral convexity of the brain. US, ultrasound.

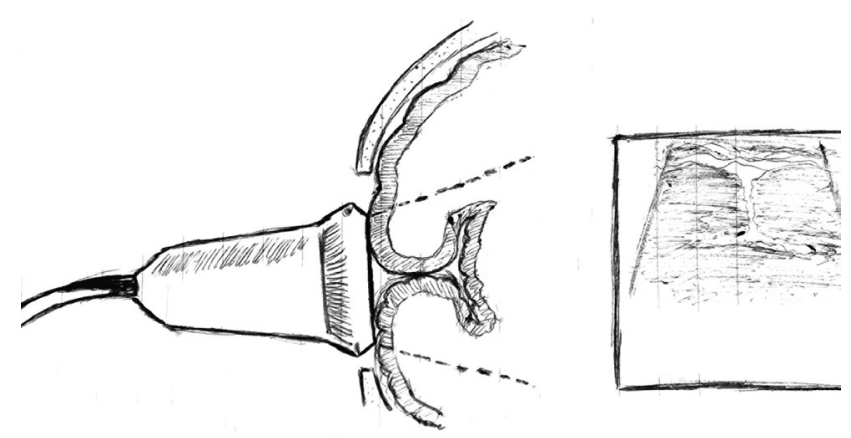

Fig. 5 Schematic representation of the position of the probe in relation to the surface of the brain to obtain a coronal view in intraoperative US,.US, ultrasound.

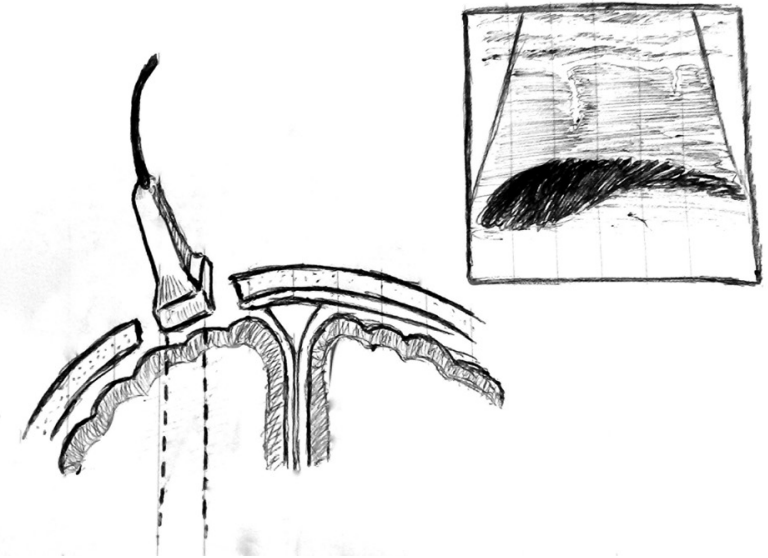

Fig. 6 Schematic representation of the position of the probe in relation to the surface of the brain to obtain a sagittal view in intraoperative US. A sagittal view on this position is recognizable by the presence of the lateral ventricle. US, ultrasound.

superficial structures, while low-frequency probes are better suited for examining deeper structures, ${ }^{11}$ for example, when assessing periventricular lesions.

Probe manipulation is another important skill that should be mastered to properly perform an intraoperative ultrasonography.

In addition to adequately placing the transducer to obtain the desired plane of view, as it was previously mentioned, there are additional movements that will help to better visualize the target lesion and improve localization.

First, depending on the anatomical site being evaluated, slight pressure on the surface can improve image quality. It affects the echogenicity of the tissue and shortens the distance to the structure of interest. ${ }^{11}$

Sometimes, the lesion is not perfectly aligned with the initial plane of view, that is, while realigning the probe on the surface may allow for a better image, this can be achieved by sliding or rotating the probe until the image seen on the screen is sufficient to proceed with the procedure.

Finally, tilting the probe may also improve the quality of the image by achieving a view of the short axis of the lesion in question, and this may also give a clue for the surgical rout.

\section{Discussion}

\section{Intraoperative Ultrasound in Neurosurgery}

The use of US for the resection of brain tumor was implemented since the 1980s; one of the first reports of tumor visibility in US was done by Le Roux et al in 1992, who described that most tumors, including high- and low-grade gliomas, were visible during surgery using common US probes. ${ }^{15}$

As it is known, one of the primary goals for tumor surgery is gross total resection (GTR) of the lesion, as it has been established that resection over $95 \%$, or even $75 \%$ of the enhanced lesion, will result in improvement in overall survival (OS) and progression-free survival (PFS). ${ }^{16-18}$

Intraoperative navigation technology has aided immensely in this field, providing better accuracy in tumor location 
and providing tumor margins to both improving grade of resection and preserving healthy tissue. These benefits can be limited however by brain-shift, in which structures change shape and position as a result of intraparenchymal swelling, gravity, tumor resection, CSF drainage, and other factors; this shift has been estimated to be as much as 11 to $12 \mathrm{~mm} .{ }^{19}$ One of the many strategies to improve accuracy during navigation is the use of intraoperative US.

Intraoperative US has been proven to be of great value in assessing tumor volume, and localization, especially for primary resection of gliomas and metastatic lesions. ${ }^{20,21}$

In addition to providing a reliable real-time image of the surgical field, intraoperative US can also be used to estimate brain-shift and provide correctional strategies to the software. $^{22}$

Petridis et al performed a retrospective study to show the importance of intraoperative US in locating tumors. Thirty-four patients who were taken into surgery for a low-grade glioma were studied. The authors reported that $100 \%$ of the tumors were located using a combination of intraoperative US and MRI neuronavigation. ${ }^{5}$

Another way that tumor localization by US is of value is the capacity to perform needle biopsies. US-guided biopsies can be performed even in deep locations because the realtime image provided will help to place the needle safely in the place of interest. ${ }^{23}$

The extent of resection (EOR) can also be improved using intraoperative US. Historically, great discrepancy has existed between surgeon estimate of the EOR and actual EOR measured by postoperative MRI, which states that the EOR is overestimated by the surgeon in most of the cases. ${ }^{24}$ This is why intraoperative aids such as US have gained much attention in the past few years.

In one study comparing assessment of residual tumor using intraoperative US and intraoperative MRI, it was revealed that intraoperative US could reliably detect up to $1 \mathrm{~cm}$ of residual tumor, and by doing so, improve the EOR. ${ }^{25}$ Another study with 45 patients concluded that US can be used to maximize EOR, given that it could detect residual tumor with high-sensitivity..$^{13}$ Even so, intraoperative US for assessing EOR should be used with care, given that many variables could affect image quality and give false imaging as a result of acoustic enhancement artifact from saline and clotted blood in the resection cavity, both of which can appear hyperechoic on US. ${ }^{26}$ Another study showed up to $89 \%$ concordance with histopathology in hyperechoic areas, which clearly extended into the isoechogenic brain parenchyma, but only $56 \%$ concordance along the hyperechoic rim of the resection cavity. ${ }^{26}$ These results show that although US is a valuable tool to improve the EOR, it should be interpreted carefully and aided by other technologies.

With regard to EOR, a recent meta-analysis that included several studies with a total of 739 patients showed an average EOR of 79\%, stating important heterogeneity among the studies. Statistical analysis showed concordance of $89 \%$ between postoperative MRI and intraoperative US, with false-positive and false-negative results of $9 \%{ }^{27}$

A second meta-analysis by Zhang et al included 37 articles, mainly developed in China, and established the sensibility and specificity for intraoperative US to detect residual tumor of $89 \%$ and $91 \%$, respectively. ${ }^{28}$

- Table 1 shows a summary of the published articles that study the impact of intraoperative US on EOR.

Recently, a study was published by Munkvold et al that found several factors associated with the capacity of intraoperative US to detect residual tumor during surgery. They determined that tumor volume and tumor depth were the main factors that influenced the sensitivity for intraoperative US to detect residual tumor; small superficial tumors being more likely to be completely resected. ${ }^{29}$

One can infer that the impact of the usage of intraoperative US on the clinical course of patients with brain tumors is related to the ability to locate and better resect these lesions.

One study attempted to show the impact of intraoperative US on the OS of patients with high- and low-grade gliomas. The overall conclusion was that the usage of intraoperative US had a positive impact on OS of patients with both highand low-grade gliomas. ${ }^{27}$

The neurosurgical applications of US will most likely vary in the future. With the development of new US transducers, contrast agents, and processing systems, this technique will most likely be used more frequently and in more ways than today.

Contrast-enhanced US is one of these new developments, and has been used widely in other fields such as surgery for the liver and kidney. ${ }^{30}$ This technique involves the injections of microbubbles that comprise an inert gas, such as perfluorocarbon or nitrogen, encapsulated in a layer of protein or polymers. These microbubbles are not affected in the lung

Table 1 Impact of intraoperative ultrasound on the extent of resection

\begin{tabular}{|c|c|c|c|}
\hline Author & Year & Number of patients & Mean \% of extent of resection \\
\hline Gerganov et al25 & 2009 & 25 & $80.8 \%$ \\
\hline Chacko et $\mathrm{al}^{26}$ & 2003 & 35 & $71.4 \%$ \\
\hline Wang et $\mathrm{al}^{29}$ & 2012 & 137 & $81.8 \%$ \\
\hline Solheim et a ${ }^{21}$ & 2010 & 142 & $74.5 \%$ \\
\hline Liang et $\mathrm{a}^{30}$ & 2013 & 80 & $86.2 \%$ \\
\hline Tian et $a^{31}$ & 2009 & 88 & $76.7 \%$ \\
\hline Sweeney et al ${ }^{32}$ & 2018 & 260 & $81 \%$ \\
\hline
\end{tabular}


circulation and are able to cross into the arterial circulation, allowing to act as a contrast agent depending on the tumor vascularity and perfusion. ${ }^{31}$ Recently, many studies have been performed to establish the role of microbubbles in the field of brain tumor surgery; significant information has been obtained by a few studies, which showed that contrast-enhanced US can be used safely and allows for superior image quality. ${ }^{32-34}$

Strain elastography is another feature of US technology that evaluates tissue macrostructure, as it compares characteristics of the ultrasound beam through tissue before and after compression, and so it is able to map tissue stiffness. Using this technology in combination with B-Mode US, a better differentiation of tumor and normal brain tissue can be achieved. ${ }^{31,35,36}$

Although intraoperative US has been proved to be a very valuable tool, given its versatility, cost-effectiveness, and efficiency, it has limitations that cannot be ignored.

As it is well known that US is very user-dependent, image quality and restricted field of view are issues that limit accurate interpretation, especially for surgeons who lack proper training. ${ }^{31}$ Small lesions may also be challenging to detect. In addition, blood and hemostatic agents within the resection cavity can confound image interpretation. ${ }^{37}$

Nevertheless, as previously mentioned, improvement in technology will lead to better image resolution and probe features; together with research into image analysis, it is anticipated that these hurdles will be overcome.

\section{Conclusion}

Intraoperative US is a very valuable tool that improves tumor location and resection in the field of neurosurgery; however, it is very important to understand the basics of US and the proper technique to obtain the benefits of this technology.

Given its versatility and cost-effectiveness, intraoperative US should be a tool that every neurosurgeon involved in the field of neuro-oncology should master to provide better care to patients.

\section{Conflict of Interest}

None declared.

\section{References}

1 Wood RW, Loomis AL. The physical and biological effects of high frequency sound waves of great intensity. Philos Mag Ser 7 1927;4(22):417-436

2 Newman PG, Rozycki GS. The history of ultrasound. Surg Clin North Am 1998;78(2):179-195

3 Reid MH. Ultrasonic visualization of a cervical cord cystic astrocytoma. AJR Am J Roentgenol 1978;131(5):907-908

4 Sosna J, Barth MM, Kruskal JB, Kane RA. Intraoperative sonography for neurosurgery. J Ultrasound Med 2005;24 (12):1671-1682

5 Petridis AK, Anokhin M, Vavruska J, Mahvash M, Scholz M. The value of intraoperative sonography in low grade glioma surgery. Clin Neurol Neurosurg 2015;131:64-68
6 Aldrich JE. Basic physics of ultrasound imaging. Crit Care Med 2007;35(5, Suppl):S131-S137

7 Falyar CR. Ultrasound in anesthesia: applying scientific principles to clinical practice. AANA J 2010;78(4):332-340

8 Tempany CM, McDannold NJ, Hynynen K, Jolesz FA. Focused ultrasound surgery in oncology: overview and principles. Radiology 2011;259(1):39-56

9 Riccabona M. Basics, principles, techniques and modern methods in paediatric ultrasonography. Eur J Radiol 2014;83 (9):1487-1494

10 Pollard BA, Chan VW. Introductory Curriculum for Ultrasound-Guided Regional Anesthesia. Toronto, Canada: University of Toronto Press Inc; 2009

11 Ihnatsenka B, Boezaart AP. Ultrasound: basic understanding and learning the language. Int J Shoulder Surg 2010;4(3):55-62

12 Ivanov M, Wilkins S, Poeata I, Brodbelt A. Intraoperative ultrasound in neurosurgery - a practical guide. $\mathrm{Br} \mathrm{J}$ Neurosurg 2010;24(5):510-517

13 Woydt M, Krone A, Becker G, Schmidt K, Roggendorf W, Roosen K. Correlation of intra-operative ultrasound with histopathologic findings after tumour resection in supratentorial gliomas. A method to improve gross total tumour resection. Acta Neurochir (Wien) 1996;138(12):1391-1398

14 Cengiz C, Keramettin A. Intraoperative ultrasonographic characteristics of malignant intracranial lesions. Neurol India 2005;53(2):208-211

15 Le Roux PD, Berger MS, Wang K, Mack LA, Ojemann GA. Low grade gliomas: comparison of intraoperative ultrasound characteristics with preoperative imaging studies. J Neurooncol 1992;13(2):189-198

16 Lacroix M, Abi-Said D, Fourney DR, et al. A multivariate analysis of 416 patients with glioblastoma multiforme: prognosis, extent of resection, and survival. J Neurosurg 2001;95(2):190-198

17 Keles GE, Chang EF, Lamborn KR, et al. Volumetric extent of resection and residual contrast enhancement on initial surgery as predictors of outcome in adult patients with hemispheric anaplastic astrocytoma. J Neurosurg 2006;105(1):34-40

18 McGirt MJ, Chaichana KL, Gathinji M, et al. Independent association of extent of resection with survival in patients with malignant brain astrocytoma. J Neurosurg 2009;110(1):156-162

19 Roberts DW, Hartov A, Kennedy FE, Miga MI, Paulsen KD. Intraoperative brain shift and deformation: a quantitative analysis of cortical displacement in 28 cases. Neurosurgery 1998;43(4):749-758

20 Hammoud MA, Ligon BL, elSouki R, Shi WM, Schomer DF, Sawaya R. Use of intraoperative ultrasound for localizing tumors and determining the extent of resection: a comparative study with magnetic resonance imaging. J Neurosurg 1996;84(5):737-741

21 Solheim O, Selbekk T, Jakola AS, Unsgård G. Ultrasound-guided operations in unselected high-grade gliomas-overall results, impact of image quality and patient selection. Acta Neurochir (Wien) 2010;152(11):1873-1886

22 Unsgaard G, Selbekk T, Brostrup Müller T, et al. Ability of navigated 3D ultrasound to delineate gliomas and metastasescomparison of image interpretations with histopathology. Acta Neurochir (Wien) 2005;147(12):1259-1269, discussion 1269

23 RubinJM, Dohrmann GJ.Use of ultrasonically guided probes and catheters in neurosurgery. Surg Neurol 1982;18(2):143-148

24 Orringer D, Lau D, Khatri S, et al. Extent of resection in patients with glioblastoma: limiting factors, perception of resectability, and effect on survival. J Neurosurg 2012;117(5):851-859

25 Gerganov VM, Samii A, Akbarian A, Stieglitz L, Samii M, Fahlbusch R. Reliability of intraoperative high-resolution 2D ultrasound as an alternative to high-field strength MR imaging 
for tumor resection control: a prospective comparative study. J Neurosurg 2009;111(3):512-519

26 Chacko AG, Kumar NK, Chacko G, Athyal R, Rajshekhar V. Intraoperative ultrasound in determining the extent of resection of parenchymal brain tumours-a comparative study with computed tomography and histopathology. Acta Neurochir (Wien) 2003;145(9):743-748

27 Mahboob S, McPhillips R, Qiu Z, et al. Intraoperative ultrasound-guided resection of gliomas: a meta-analysis and review of the literature. World Neurosurg 2016;92:255-263

28 Zhang G, Li Z, Si D, Shen L. Diagnostic ability of intraoperative ultrasound for identifying tumor residual in glioma surgery operation. Oncotarget 2017;8(42):73105-73114

29 Munkvold BKR, Jakola AS, Reinertsen I, Sagberg LM, Unsgård G, Solheim $\mathrm{O}$. The diagnostic properties of intraoperative ultrasound in glioma surgery and factors associated with gross total tumor resection. World Neurosurg 2018;115:e129-e136

30 Denham SL, Alexander LF, Robbin ML. Contrast-enhanced ultrasound: practical review for the assessment of hepatic and renal lesions. Ultrasound Q 2016;32(2):116-125

31 Bal J, Camp SJ, Nandi D. The use of ultrasound in intracranial tumor surgery. Acta Neurochir(Wien) 2016;158(6):1179-1185
32 Prada F, Perin A, Martegani A, et al. Intraoperative contrast-enhanced ultrasound for brain tumor surgery. Neurosurgery 2014; 74(5):542-552

33 Wu DF, He W, Lin S, Han B, Zee CS. Using real-time fusion imaging constructed from contrast-enhanced ultrasonography and magnetic resonance imaging for high-grade glioma in neurosurgery. World Neurosurg 2019;125:e98-e109

34 Mattei L, Prada F, Legnani FG, Perin A, Olivi A, DiMeco F. Neurosurgical tools to extend tumor resection in hemispheric low-grade gliomas: conventional and contrast enhanced ultrasonography. Childs Nerv Syst 2016;32(10):1907-1914

35 Chauvet D, Imbault M, Capelle L, et al. In vivo measurement of brain tumour elasticity using intraoperative shear wave elastography. Ultraschall Med 2016;37(6):584-590

36 Chakraborty A, Bamber JC, Dorward NL. Preliminary investigation into the use of ultrasound elastography during brain tumour resection. Ultrasound 2012;20(1):33-40

37 Selbekk T, Jakola AS, Solheim O, et al. Ultrasound imaging in neurosurgery: approaches to minimize surgically induced image artefacts for improved resection control. Acta Neurochir (Wien) 2013;155(6):973-980 